\title{
The Application of Immunocytochemistry to Direct Smears in the Diagnosis of Effusions
}

Stewart M. Knoepp, M.D., Ph.D., ${ }^{\dagger}$ Jeremiah Placido, M.D., ${ }^{\dagger}$ Kristina L. Fields, B.S., Dafydd Thomas, M.D., Ph.D., and Michael H. Roh, M.D., Ph.D. ${ }^{*}$

\begin{abstract}
Metastatic malignancy represents a common cause of effusions. Immunocytochemistry (ICC) is useful in confirming malignancy and gaining insight into the site of origin. Cell blocks are commonly utilized for this purpose; nonetheless, when the malignant cells are sparse, they may not be represented in cell blocks thereby precluding immunophenotypic characterization. Thus, we sought to investigate the utility of direct smear preparations as a platform for ICC in the diagnosis of effusions. Air-dried, unstained direct smears were prepared from 49 malignant effusions and 17 reactive effusions for comparison. ICC for EMA and MOC-31 highlighted the tumor cells in 91 and $98 \%$ of the malignant effusions tested, respectively. EMA immunoreactivity was focally observed within the calretinin-positive mesothelial cell population in $1(6 \%)$ of the 17 reactive effusions. ICC for MOC31 was negative in all reactive effusions. Site-specific immunomarkers were also evaluated. Immunoreactivity for Napsin-A and TTF-1 were observed in 78 and $67 \%$ of metastatic lung adenocarcinomas, respectively. ICC for PAX8 highlighted metastatic Müllerian and thyroid carcinomas in 100\% of cases tested. CDX-2 immunoreactivity was observed in 25,60 , and $100 \%$ of metastatic gastric, pancreatic, and colorectal adenocarcinomas, respectively. Positivity for p63 was observed in $75 \%$ of metastatic urothelial cell carcinomas and the one case of pulmonary squamous cell carcinoma examined. Calretinin ICC highlighted the tumor cells in both malignant mesothelioma cases tested as well as the benign mesothelial cells in the reactive effusions. In conclusion, direct smears represent an effective platform for the performance of ICC in the diagnosis of malignant effusions. Diagn. Cytopathol. 2013;41:425-430. @ 2012 Wiley Periodicals, Inc.
\end{abstract}

Department of Pathology, University of Michigan Medical School, Ann Arbor, Michigan

${ }^{\dagger}$ Dr. Knoepp and Dr. Placido contributed equally to the work.

*Correspondence to: Michael H. Roh, MD, PhD, $2 \mathrm{G} 332 \mathrm{UH}$, Department of Pathology, University of Michigan Health System, 1500 E. Medical Center Drive, Ann Arbor, Michigan 48109, USA.

E-mail: mikro@med.umich.edu

Received 18 November 2011; Accepted 11 February 2012

DOI $10.1002 / \mathrm{dc} .22852$

Published online 30 April 2012 in Wiley Online Library (wileyonlinelibrary.com).
Key Words: effusion; carcinoma; immunocytochemistry; direct smear; cytology

Metastatic malignancy is a common cause of fluid accumulation in the pleural, peritoneal, and pericardial spaces. Of these, metastatic carcinoma represents the most common etiology of malignant effusions. ${ }^{1}$ Clinical information including the site of effusion, sex of the patient, and any history of malignancy in addition to knowledge of commonly encountered malignant effusions can be of aid to the pathologist during the initial evaluation and accurate diagnosis of an effusion specimen. ${ }^{2,3}$ Ancillary immunocytochemistry (ICC) can be employed to confirm a metastasis from a patient's already documented primary malignancy. In addition, for instances in which a malignancy is suspected in an effusion specimen and the primary site is unknown, immunocytochemistry can serve as a diagnostically useful adjunct to routine cytomorphologic evaluation as it can assist in identifying malignant cells and elucidating possible sites of origin. Accurate and specific diagnoses of malignant effusions yield important information regarding the nature and extent of metastatic disease enabling prompt, appropriate treatment.

In this regard, cell block preparations are commonly utilized for ancillary immunohistochemical studies. However, this platform is fraught with a significant limitation; it is not uncommon for the cells of interest to present only as a minority population within the milieu of background mesothelial cells, histiocytes, and other inflammatory cells. For cases in which the malignant cells are sparse in cytology specimens, the cells of interest may not be well represented in cell blocks thereby precluding further characterization via ancillary techniques. As an illustration, Mulvahy analyzed 75 malignant peritoneal washing specimens; malignant cells were visualized on smear preparations in $97 \%$ of these cases while they were seen in the cell block preparations in $51 \%$ of cases. ${ }^{4}$ 
Furthermore, we and others have observed variations in tumor cellularity in cell block preparations of lung carcinoma cytology specimens. ${ }^{5,6}$ In our previous analysis of 26 lung carcinoma cytology specimens, cell blocks were acellular in $9(35 \%)$ of these cases. ${ }^{5}$ Similarly, Swati et al. observed that $2(25 \%)$ of 8 cell blocks from lung cancer aspirates exhibited a percent tumor cellularity of $2 \%$ or less. ${ }^{6}$

We have previously demonstrated that direct smears represent a robust platform for the performance of ICC and molecular testing using cytology specimens. For instance, we reported the application of ICC for NapsinA, TTF-1, and p63 to unstained, air-dried direct smears of pulmonary non-small cell carcinomas to assist in the subclassification of these tumors as adenocarcinoma or squamous cell carcinoma. ${ }^{7}$ Next, we demonstrated that DiffQuik stained direct smears of lung cancers can be successfully utilized for tumor cell microdissection, subsequent tumor DNA extraction, and molecular testing for EGFR and KRAS mutations. ${ }^{5}$ In addition, we extended our methodology to fine-needle aspirates of metastatic melanoma. Specifically, unstained air-dried direct smears were utilized for confirmatory ICC using antibodies against S100, HMB45, and Melan-A. ${ }^{8}$ Furthermore, the effectiveness of Diff-Quik stained direct smears of melanoma as a platform for BRAF mutational analysis was demonstrated in that study. Recently, we reported the efficacy of ICC for CK20 using direct smears in the diagnosis of metastatic Merkel cell carcinoma. ${ }^{9}$

There are only a limited number of studies in the literature that address the application of ICC to direct smears prepared from effusions. ${ }^{10-12}$ Thus, we sought to further investigate the applicability of ICC to direct smears prepared from malignant and benign effusion specimens. Herein, we report our experience with the application of ICC for EMA and MOC-31 in the diagnosis of malignant effusions as well as the use of select differentiation- and site-specific immunomarkers geared towards ascertaining the site of origin of malignant effusions.

\section{Materials and Methods}

The study was approved by the Institutional Review Board at University of Michigan. Air-dried, unstained direct smears were prepared using positively charged slides for 49 malignant effusions. Specifically, 50-100 $\mathrm{cm}^{3}$ of each fluid specimen was centrifuged for $5 \mathrm{~min}$ at $2,400 \mathrm{rpm}$ at room temperature. The supernatant was carefully decanted and the pellet was resuspended in residual supernatant fluid using a syringe and evenly distributed onto slides for smear preparation. One smear was stained with Diff-Quik for cytomorphologic analysis as a reference for identifying cytologically malignant cells on the immunostained slides. For each case, the electronic
Table I. Results of Immunocytochemistry on Direct Smears Prepared From Malignant Effusions

\begin{tabular}{|c|c|c|}
\hline Primary site (number of cases) & Immunostain & $\begin{array}{l}\text { No. of positive } \\
\text { (\% of cases) }\end{array}$ \\
\hline \multirow[t]{4}{*}{ Lung adenocarcinoma $(n=9)$} & EMA & $9(100 \%)$ \\
\hline & MOC-31 & $9(100 \%)$ \\
\hline & Napsin-A & $7(78 \%)$ \\
\hline & TTF-1 & $6(67 \%)$ \\
\hline Lung squamous cell carcinoma $(n=1)$ & p63 & $1(100 \%)$ \\
\hline \multirow[t]{2}{*}{ Breast adenocarcinoma $(n=4)$} & EMA & $4(100 \%)$ \\
\hline & MOC-31 & $4(100 \%)$ \\
\hline \multirow[t]{3}{*}{ Gastric adenocarcinoma $(n=4)$} & EMA & $3(75 \%)$ \\
\hline & MOC-31 & $4(100 \%)$ \\
\hline & CDX-2 & $1(25 \%)$ \\
\hline \multirow[t]{3}{*}{ Pancreatic adenocarcinoma $(n=5)$} & EMA & $3(60 \%)$ \\
\hline & MOC-31 & $5(100 \%)$ \\
\hline & $\mathrm{CDX}-2$ & $3(60 \%)$ \\
\hline \multirow[t]{3}{*}{ Colorectal adenocarcinoma $(n=2)$} & EMA & $1(50 \%)$ \\
\hline & MOC-31 & $2(100 \%)$ \\
\hline & $\mathrm{CDX}-2$ & $2(100 \%)$ \\
\hline \multirow[t]{3}{*}{ Müllerian adenocarcinoma ( $n=15)$} & EMA & $15(100 \%)$ \\
\hline & MOC-31 & $15(100 \%)$ \\
\hline & PAX8 & $15(100 \%)$ \\
\hline Urothelial cell carcinoma $(n=4)$ & p63 & $3(75 \%)$ \\
\hline \multirow[t]{3}{*}{ Malignant mesothelioma $(n=2)$} & EMA & $2(100 \%)$ \\
\hline & MOC-31 & $1(50 \%)$ \\
\hline & Calretinin & $2(100 \%)$ \\
\hline \multirow[t]{4}{*}{ Papillary thyroid carcinoma $(n=1)$} & EMA & $1(100 \%)$ \\
\hline & MOC-31 & $1(100 \%)$ \\
\hline & TTF-1 & $1(100 \%)$ \\
\hline & PAX8 & $1(100 \%)$ \\
\hline \multirow[t]{4}{*}{ Anaplastic thyroid carcinoma $(n=1)$} & EMA & $1(100 \%)$ \\
\hline & MOC-31 & $1(100 \%)$ \\
\hline & TTF-1 & $0(0 \%)$ \\
\hline & PAX8 & $1(100 \%)$ \\
\hline \multirow{7}{*}{$\begin{array}{l}\text { Adenocarcinoma, unknown } \\
\quad \text { primary }(n=1)\end{array}$} & EMA & $1(100 \%)$ \\
\hline & MOC-31 & $1(100 \%)$ \\
\hline & Napsin-A & $0(0 \%)$ \\
\hline & TTF-1 & $0(0 \%)$ \\
\hline & PAX8 & $0(0 \%)$ \\
\hline & CDX-2 & $0(0 \%)$ \\
\hline & Calretinin & $0(0 \%)$ \\
\hline
\end{tabular}

medical record, including pathology reports and clinical notes, were examined to identify patients with known cancer histories.

ICC was performed on air-dried, unstained direct smears following formalin fixation for $60 \mathrm{~min}$. ICC for Napsin-A, TTF-1, and p63 were performed as described previously. ${ }^{7}$ ICC for EMA (1:100 dilution; DAKO, Carpenteria, CA), MOC-31 (1:100 dilution; DAKO, Carpenteria CA), PAX8 (1:200 dilution; Protein Tech, Chicago, IL), CDX-2 (predilute; Ventana Medical Systems, Tucson, AZ), and calretinin (1:100 dilution; Zymed, Carlsbad, CA) was performed following pretreatment in CC1 buffer (Ventana Medical Systems, Tucson, AZ). The list of antibodies tested for each category of malignant effusions based on the type and origin of malignancy is shown in Table I.

For each antibody, immunocytochemistry performed on unstained smears prepared from specimens with established diagnoses, in which positive immunostaining 
results were obtained, represented positive controls. Furthermore, immunohistochemistry performed on unstained sections from tissue microarrays represented an additional source of positive controls. Negative control immunostains, in which the primary antibody was omitted, were also performed on unstained direct smears for each case.

Next, air-dried, unstained direct smears were prepared on positively charged slides for 17 reactive effusions. In these 17 cases, ICC for all eight antibodies was performed; this cohort served as a negative control cohort as well.

\section{Results}

Of the 49 malignant effusions examined in this study, the primary site was known in 48 cases (Table I): 10 lung carcinomas (9 adenocarcinomas and 1 squamous cell carcinoma); 4 breast adenocarcinomas; 4 gastric adenocarcinomas; 5 pancreatic adenocarcinomas; 2 colorectal adenocarcinomas; 15 Müllerian adenocarcinomas; 4 urothelial cell carcinomas; 2 malignant mesotheliomas; and 2 thyroid carcinomas (1 papillary thyroid carcinoma and 1 anaplastic thyroid carcinoma). In one case of malignancy, the site of origin was unknown and immunocytochemical workup failed to identify a definite primary site. This patient was considered clinically to harbor a tumor of unknown primary. For comparison, 17 cases of benign, reactive effusions were also examined in this study.

ICC on direct smears for EMA and MOC-31 were performed for 44 of the 49 malignant effusions (Table I); the 1 case of metastatic pulmonary squamous cell carcinoma and the 4 cases of metastatic urothelial cell carcinoma were not examined by ICC for these two markers. Strong, diffuse immunoreactivity for EMA was observed in the tumor cells in $91 \%$ (40/44) of the cases tested (Fig. 1). Specifically, $100 \%$ of the lung adenocarcinomas (9/9), breast adenocarcinomas (4/4), Müllerian adenocarcinomas $(15 / 15)$, malignant mesotheliomas $(2 / 2)$, and thyroid carcinomas $(2 / 2)$ were positive for EMA. Furthermore, $75 \%$ (3/4) of gastric adenocarcinomas, $60 \%$ (3/5) of pancreatic adenocarcinomas, and $50 \%(1 / 2)$ of colorectal adenocarcinomas exhibited EMA positivity in the tumor cells. The one adenocarcinoma of unknown primary was positive for EMA. Next, MOC-31 immunoreactivity in the tumor cells was observed in 98\% (43/44) of the cases tested (Fig. 1). In 42 of the 43 MOC-31(+) cases, MOC-31 positivity was strong and diffuse. In one case of malignant mesothelioma, focal $(<10 \%$ of tumor cells) positivity for MOC31 was observed (Fig. 1). Of note, in the one case of gastric adenocarcinoma, two cases of pancreatic adenocarcinoma, and the one case of colorectal adenocarcinoma in which the tumor cells were negative for EMA, ICC for MOC-31 highlighted the tumor cell population. No MOC31 immunoreactivity was observed in any of the tumor cells in one case of malignant mesothelioma.

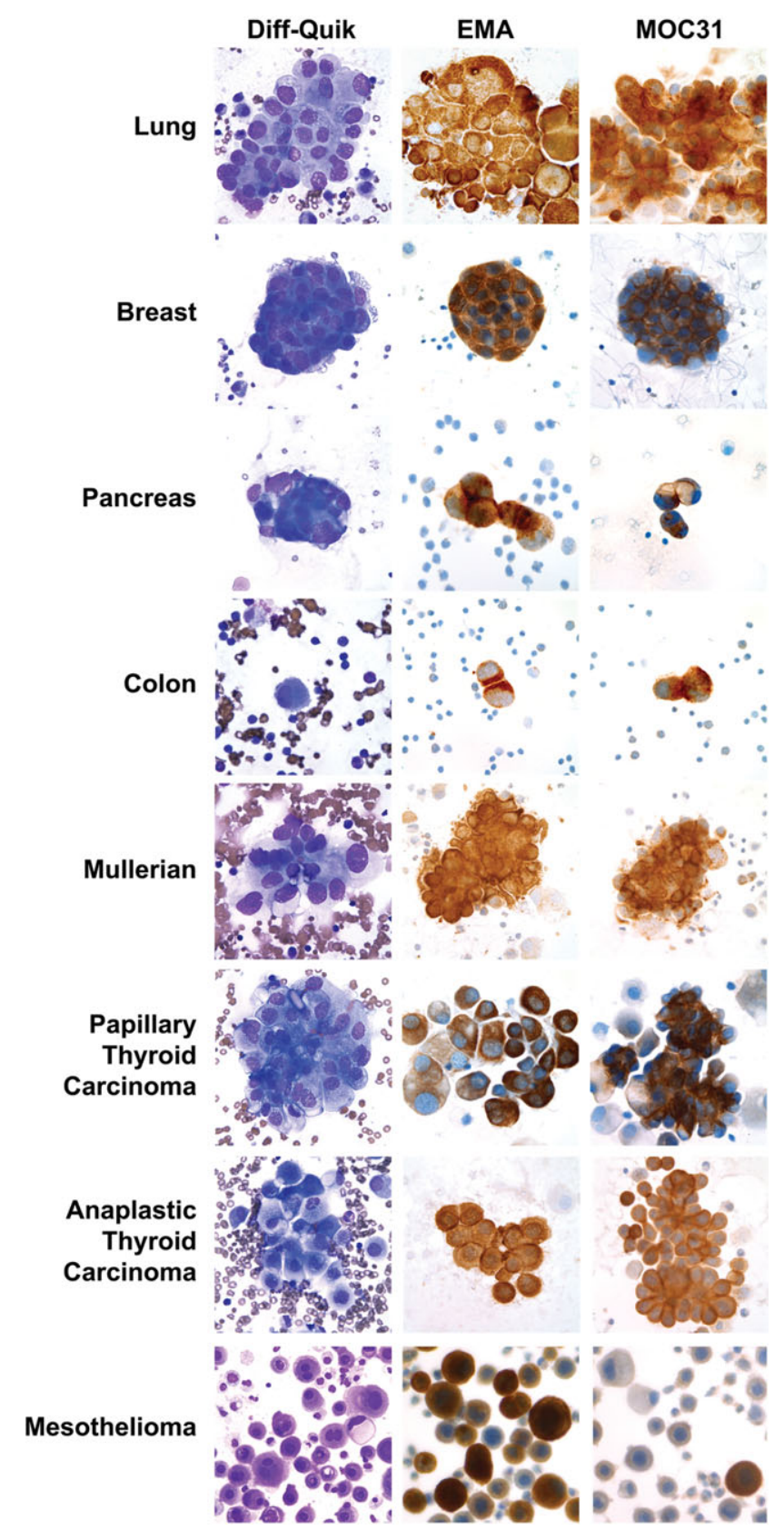

Fig. 1. Immunocytochemistry for EMA and MOC-31 using direct smears of malignant effusions. Photomicrographs of Diff-Quik stained smears and immunostains for EMA and MOC-31 for representative cases of metastatic lung adenocarcinoma, breast adenocarcinoma, pancreatic adenocarcinoma, colonic adenocarcinoma, Müllerian adenocarcinoma, papillary thyroid carcinoma, anaplastic thyroid carcinoma, and malignant mesothelioma are displayed $(\times 1,000)$. [Color figure can be viewed in the online issue, which is available at wileyonlinelibrary.com.]

Next, we examined the use of site-specific immunomarkers utilizing direct smears of effusions. The results are summarized in Table I and representative images are displayed in Figure 2. Immunoreactivity for Napsin-A and TTF-1 was observed in the tumor cells in $78 \%(7 / 9)$ and $67 \%(6 / 9)$ cases of lung adenocarcinoma, respectively. A 


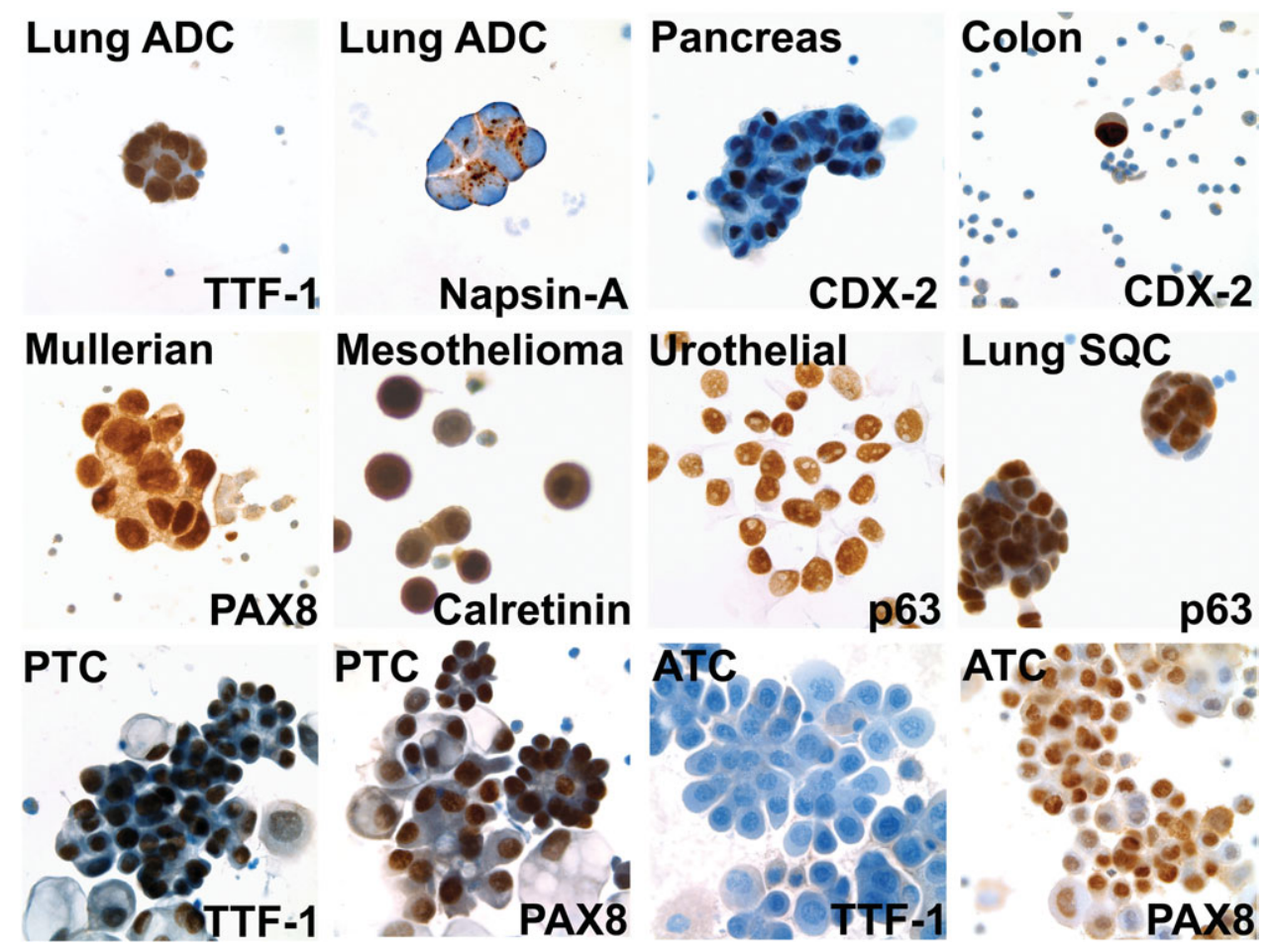

Fig. 2. Immunocytochemistry for site-specific markers using direct smears of malignant effusions. Representative immunostains for TTF-1, Napsin-A, CDX-2, PAX8, calretinin, and p63 are shown as indicated in each photomicrograph $(\times 1,000)$. Abbreviations: ADC, adenocarcinoma; SQC, squamous cell carcinoma; PTC, papillary thyroid carcinoma; ATC, anaplastic thyroid carcinoma. [Color figure can be viewed in the online issue, which is available at wileyonlinelibrary.com.]

granular cytoplasmic staining pattern for Napsin-A and distinct nuclear immunopositivity for TTF-1 was observed. Nuclear immunoreactivity for CDX-2 was observed in $25 \%(1 / 4)$ of gastric adenocarcinomas, $60 \%$ $(3 / 5)$ of pancreatic adenocarcinomas, and $100 \%(2 / 2)$ of colorectal adenocarcinomas. ICC for PAX8 highlighted the nuclei of the tumor cells in $100 \%(15 / 15)$ of Müllerian adenocarcinomas and 100\% (2/2) of thyroid carcinomas. Nuclear immunoreactivity for p63 was observed in the one case of pulmonary squamous cell carcinoma and $75 \%$ (3/4) of urothelial cell carcinomas. Nuclear and cytoplasmic immunoreactivity for calretinin was observed in $100 \%(2 / 2)$ of cases of malignant mesothelioma, confirming mesothelial differentiation. In the one adenocarcinoma of unknown primary, none of the site-specific markers tested (Table I) were positive. For the above cases, the negative control immunostains, in which the primary antibody was omitted, were negative confirming the specific staining patterns observed for each antibody.

A total of 17 benign, reactive effusions were also tested for comparison. Calretinin positivity was observed in the mesothelial cell population in all cases. Focal EMA positivity $(<10 \%)$ was observed in the mesothelial cells in $6 \%(1 / 17)$ of reactive effusions. Immunostains for MOC31, Napsin-A, TTF-1, CDX-2, PAX8, and p63 were negative in all 17 cases of benign effusions.

\section{Discussion}

Traditionally, cell blocks are utilized for immunophenotyping tumor cells in malignant effusions. Nonetheless, paucicellularity of the tumor cell population and, consequently, underrepresentation of tumor cells in cell block preparations represents a significant limitation. ${ }^{4-8}$ Employing direct smears as a platform for immunocytochemistry is advantageous in several respects. First, multiple unstained direct smears can be prepared from pelleted cellular material. One of the smears can be rapidly stained via the Diff-Quik staining protocol and immediately assessed for the presence or absence of tumor cells as well as percent tumor cellularity. Second, the tumor cellularity in cell block preparations is not immediately known. Instead, the composition of cell blocks can only be ascertained after processing of the cell blocks and subsequent preparation of $H \& E$ slides by histotechnologists. This leads to a relative increase in turnaround time for rendering an accurate, specific diagnosis encompassing requisite immunostains. In contrast, unstained, air-dried direct smears can be rapidly prepared, immediately submitted for immunocytochemistry without the need for interim processing steps, and the immunostained slides can be obtained within hours. Third, the cytomorphology of the tumor cells on the immunostains are preserved facilitating direct correlation with Diff-Quik stained smears. 
There currently exist a limited number of studies examining the use of a panel of immunocytochemical stains to direct smears prepared from effusions. ${ }^{10-12}$ Herein, we report our experience in applying ICC to direct smear preparations of effusion specimens. Our study augments the current knowledge about this topic in several respects. Similar to our study, Ueda et al. examined ICC using antibodies directed against EMA and MOC- $31^{12}$; we observed that EMA and MOC-31 were highly sensitive in detecting malignant cells consistent with their findings. In contrast to their study, we also examined the use of additional markers that are specific to particular primary sites of origin. Similar to Pomjanski et al., ${ }^{10}$ we investigated the application of ICC for the site-specific markers, TTF1 and CDX-2 to direct smears. Furthermore, we extended our analysis to investigating additional site-specific markers, specifically PAX8, Napsin-A, and p63. Our analysis also differs from the two aforementioned studies with respect to the nature of direct smear preparations used. Ueda et al and Pomjanski et al. examined smears fixed in ethanol and Delaunay's fixative $(500 \mathrm{~mL} \mathrm{100 \%}$ alcohol, $500 \mathrm{~mL}$ acetone, and 10 drops of $1 M$ trichlorvinegar acid). On the other hand, we applied ICC to air-dried smears fixed in formalin. These fixation conditions recapitulate those used on histologic sections prepared from formalin-fixed tissue blocks and we have reported the successful implementation of this methodology in prior reports. $^{7-9}$

Our findings support the effectiveness of utilizing immunocytochemical stains for EMA and MOC-31 in the diagnosis of malignant effusions. ICC for EMA and MOC-31 highlighted the tumor cells in 91 and $98 \%$ of malignant effusions tested, respectively. In contrast, the immunostains for EMA and MOC-31 were negative in 94 and $100 \%$ of the benign, reactive effusions tested. This high level of sensitivity and specificity are concordant with the results of previous studies that evaluated these two markers in the diagnosis of effusions. ${ }^{13-16}$ Of note, both cases of malignant mesothelioma examined in our study exhibited EMA and calretinin positivity reinforcing the conclusion from other studies that these two markers can be utilized in the diagnosis of malignant mesothelioma in effusions. ${ }^{13,16-19}$ On the direct smear preparations, the EMA immunostain appeared to highlight the malignant mesothelioma cell population in a diffuse cytoplasmic pattern (Fig. 1). It should be noted that this staining pattern can be observed in the context of plasma membrane staining as entire, intact cells rather than crosssections of cells are being examined on immunostains of direct smears. In support of this, thick membranous immunoreactivity for EMA in the malignant cells was observed on the immunohistochemical stains performed on the corresponding cell block sections for both cases (not shown).
In addition, we examined the application of ICC for a panel of site-specific markers to direct smears prepared from effusions. First, immunoreactivity for Napsin-A and TTF-1 was observed in 78 and $67 \%$ of pulmonary adenocarcinomas. These results recapitulate the findings of two prior studies that demonstrated the increased sensitivity of ICC for Napsin-A, compared to TTF-1, in the detection of metastatic lung adenocarcinoma in effusion specimens. $^{20,21}$ Second, we observed that PAX8 was highly effective in detecting metastatic carcinomas of Müllerian and thyroid origin, consistent with the findings of prior studies. $^{22-24}$ Specifically, PAX8 immunoreactivity was seen in the tumor cells in all Müllerian and thyroid carcinomas examined. Interestingly, the one case of metastatic anaplastic thyroid carcinoma examined in our study exhibited a TTF-1(-)/PAX8(+) immunophenotype. This is in line with the findings of Nonaka et al. and Bishop et al. which demonstrated the increased sensitivity of ICC for PAX8, compared to TTF-1, in the detection of anaplastic thyroid carcinoma. ${ }^{25,26}$ Third, p63 is expressed in a high proportion of squamous cell carcinomas as well as urothelial cell carcinomas. ${ }^{7,27}$ Accordingly, in this study, we observed p63 expression in metastatic squamous cell carcinoma $(1 / 1 ; 100 \%)$ and urothelial cell carcinoma $(3 / 4$; $75 \%$ ) in effusions. Finally, we observed CDX-2 immunoreactivity in 25,60 , and $100 \%$ of metastatic gastric, pancreatic, and colorectal adenocarcinomas, respectively. These rates of CDX-2 positivity in these cancers are concordant with those reported in previously published series. $^{10,28}$

In conclusion, we have demonstrated that direct smear preparations of effusions represent a robust source of cellular material and an effective platform for ICC. Appropriate panels of immunostains can be rapidly applied, in this setting, to facilitate the identification of metastatic tumor cells in malignant effusions as well as the confirmation and determination of the primary sites of origin.

\section{References}

1. Pereira TC, Saad RS, Liu Y, Silverman JF. The diagnosis of malignancy in effusion cytology: A pattern recognition approach. Adv Anat Pathol 2006;13:174-184.

2. Monte SA, Ehya H, Lang WR. Positive effusion cytology as the initial presentation of malignancy. Acta Cytol 1987;31:448-452.

3. Murphy WM, Ng AB. Determination of primary site by examination of cancer cells in body fluids. Am J Clin Pathol 1972;58: 479-488.

4. Mulvahy N. Cytohistologic correlation in malignant peritoneal washings. Analysis of 75 malignant fluids. Acta Cytol 1996;40:1231-1239.

5. Betz BL, Roh MH, Weigelin HC, et al. The application of molecular diagnostic studies interrogating EGFR and KRAS mutations to stained cytologic smears of lung carcinoma. Am J Clin Pathol 2011;136:564-571.

6. Swati I, Zhang S, Tull J, Khurana KK. Accuracy of cytology specimen and needle core biopsies for detection of KRAS mutation in non-small cell carcinoma: Comparison with resection specimen. World J Oncol 2011;2:275-280. 
7. Roh MH, Schmidt L, Placido J, et al. The application and diagnostic utility of immunocytochemistry on direct smears in the diagnosis of pulmonary adenocarcinoma and squamous cell carcinoma. Diagn Cytopathol 2011 (in press).

8. Hookim K, Roh MH, Willman J, et al. Application of immunocytochemistry and BRAF mutational analysis to direct smears of metastatic melanoma. Cancer Cytopathol 2012;120:52-61.

9. Knoepp SM, Hookim K, Placido J, Fields KL, Roh MH. The application of immunocytochemistry to cytologic direct smears of metastatic Merkel cell carcinoma. Diagn Cytopathol (in press).

10. Pomjanski N, Grote HJ, Doganay P, Schmiemann V, Buckstegge B, Bocking A. Immunocytochemical identification of carcinomas of unknown primary in serous effusions. Diagn Cytopathol 2005;33:309-315.

11. Sato A, Torii I, Okamura Y, et al. Immunocytochemistry of CD146 is useful to discriminate between malignant pleural mesothelioma and reactive mesothelium. Mod Pathol 2010;23:1458-1466.

12. Ueda J, Iwata T, Ono M, Takahashi M. Comparison of three cytologic preparation methods and immunocytochemistries to distinguish adenocarcinoma cells from reactive mesothelial cells in serous effusion. Diagn Cytopathol 2006;34:6-10.

13. Ikeda K, Tate G, Suzuki T, Kitamura T, Mitsuya T. Diagnostic usefulness of EMA, IMP3, and GLUT-1 for the immunocytochemical distinction of malignant cells from reactive mesothelial cells in effusion cytology using cytospin preparations. Diagn Cytopathol 2011; 39:395-401.

14. Kundu UR, Krishnamurthy S. Use of the monoclonal antibody MOC-31 as an immunomarker for detecting metastatic adenocarcinoma in effusion cytology. Cancer Cytopathol 2011;119:272-278.

15. Morgan RL, De Young BR, McGaughy VR, Niemann TH. MOC-31 aids in the differentiation between adenocarcinoma and reactive mesothelial cells. Cancer 1999;87:390-394.

16. Shen J, Pinkus GS, Deshpande V, Cibas ES. Usefulness of EMA, GLUT-1, and XIAP for the cytologic diagnosis of malignant mesothelioma in body cavity fluids. Am J Clin Pathol 2009;131:516-523.

17. Shield PW, Koivurinne K. The value of calretinin and cytokeratin $5 / 6$ as markers for mesothelioma in cell block preparations of serous effusions. Cytopathology 2008;19:218-223.

18. Su XY, Li GD, Liu WP, Xie B, Jiang YH. Cytological differential diagnosis among adenocarcinoma, epithelial mesothelioma, and reactive mesothelial cells in serous effusions by immunocytochemistry. Diagn Cytopathol 2011;39:900-908.

19. Hasteh F, Lin GY, Weidner N, Michael CW. The use of immunohistochemistry to distinguish reactive mesothelial cells from malignant mesothelioma in cytologic effusions. Cancer Cytopathol 2010; 118:90-96.

20. Dejmek A, Naucler P, Smedjeback A, et al. Napsin A (TA02) is a useful alternative to thyroid transcription factor-1 (TTF-1) for the identification of pulmonary adenocarcinoma cells in pleural effusions. Diagn Cytopathol 2007;35:493-497.

21. Kim JH, Kim YS, Choi YD, Lee JS, Park CS. Utility of napsin A and thyroid transcription factor 1 in differentiating metastatic pulmonary from non-pulmonary adenocarcinoma in pleural effusion. Acta Cytol 2011;55:266-270.

22. McKnight R, Cohen C, Siddiqui MT. Utility of paired box gene 8 (PAX8) expression in fluid and fine-needle aspiration cytology: An immunohistochemical study of metastatic ovarian serous carcinoma. Cancer Cytopathol 2010;118:298-302.

23. Tong GX, Devaraj K, Hamele-Bena D, et al. Pax8: A marker for carcinoma of Mullerian origin in serous effusions. Diagn Cytopathol 2011;39:567-574.

24. Wiseman W, Michael CW, Roh MH. Diagnostic utility of PAX8 and PAX2 immunohistochemistry in the identification of metastatic Mullerian carcinoma in effusions. Diagn Cytopathol 2011;39: 651-656.

25. Bishop JA, Sharma R, Westra WH. PAX8 immunostaining of anaplastic thyroid carcinoma: A reliable means of discerning thyroid origin for undifferentiated tumors of the head and neck. Hum Pathol 2011;42:1873-1877.

26. Nonaka D, Tang Y, Chiriboga L, Rivera M, Ghossein R. Diagnostic utility of thyroid transcription factors Pax8 and TTF-2 (FoxE1) in thyroid epithelial neoplasms. Mod Pathol 2008;21:192-200.

27. Buza N, Cohen PJ, Pei H, Parkash V. Inverse p16 and p63 expression in small cell carcinoma and high-grade urothelial cell carcinoma of the urinary bladder. Int J Surg Pathol 2010;18:94-102.

28. Kim JH, Choi YD, Lee JS, Lee JH, Nam JH, Choi C. Utility of thyroid transcription factor- 1 and $\mathrm{CDX}-2$ in determining the primary site of metastatic adenocarcinomas in serous effusions. Acta Cytol 2010;54:277-282. 